\title{
Bosonization Solution of the Falicov-Kimball Model
}

\author{
P. M. R. Brydon and M. Gulácsi \\ Department of Theoretical Physics, Institute of Advanced Studies, The Australian National University, Canberra, ACT 0200, Australia
} (Received 9 June 2005; published 25 January 2006)

\begin{abstract}
We use a novel approach to analyze the one-dimensional spinless Falicov-Kimball model. We derive a simple effective model for the occupation of the localized orbitals which clearly reveals the origin of the known ordering. Our study is extended to a quantum model with hybridization between the localized and itinerant states: We find a crossover between the well-known weak- and strong-coupling behaviors. The existence of electronic polarons at intermediate coupling is confirmed. A phase diagram is presented and discussed in detail.
\end{abstract}

DOI: 10.1103/PhysRevLett.96.036407

The Falicov-Kimball model (FKM) is one of the simplest yet most versatile models of strongly correlated electron systems. It describes a band of conduction (c) electrons interacting via a repulsive contact potential $G$ with localized $f$ electrons of energy $\boldsymbol{\epsilon}_{f}$. Originally conceived as a model for valence transitions (VTs) [1], the FKM is today usually regarded as a simple model of a binary alloy, the so-called crystallization problem (CP) [2]. The CP assumes fixed $c$ - and $f$-electron populations, examining the diagonal long-range order (LRO) adopted by the $f$ electrons in the ground state. Despite detailed numerical maps of the one-dimensional (1D) FKM's phase diagram [3], its behavior is rigorously understood only at weak [4] and strong coupling [5].

A $c-f$ hybridization term is usually added to the FKM in order to model mixed-valence systems [6]. The presence of quantum valence fluctuations makes this a fundamentally different problem to the FKM: It is referred to as the extended or quantum Falicov-Kimball model (QFKM). In contrast to the FKM, the QFKM's behavior remains largely unknown. Although there is no consensus on the nature of the ground state $[7,8]$, excitonic effects are expected to dominate the physics, and so this remains an important open question in the theory of optical properties of strongly correlated electron systems.

Using the well-known nonperturbative technique of bosonization, we obtain for the first time a complete theoretical description of the 1D FKM below half-filling. This analysis reveals the role of the $c$ electrons in generating the observed $f$ order; our approach naturally explains the $\mathrm{CP}$ results. We derive an effective Hamiltonian for the $f$-orbital occupancy which accurately predicts the FKM's phase diagram. For a small hybridization potential, our approach is also suitable for the QFKM. We present a phase diagram that interpolates between the weak- and strong-coupling limits; our work rigorously establishes the relevance of electronic polaron effects.

The FKM for spinless fermions has the Hamiltonian

$$
\mathcal{H}_{\mathrm{FKM}}=-t \sum_{\langle i, j\rangle} c_{i}^{\dagger} c_{j}+\epsilon_{f} \sum_{j} n_{j}^{f}+G \sum_{j} n_{j}^{c} n_{j}^{f} .
$$

PACS numbers: 71.10.Fd, 71.27.+a, 71.28.+d, 71.30.+h

We consider only repulsive potentials, $G>0$. The concentration of electrons is fixed at $n=\frac{1}{N} \sum_{j}\left\{n_{j}^{f}+n_{j}^{c}\right\}<1$, where $N$ is the number of sites. In our study of the QFKM, we adopt the standard on-site hybridization potential $\mathcal{H}_{\text {hyb }}=V \sum_{j}\left\{f_{j}^{\dagger} c_{j}+\right.$ H.c. $\}$.

Proceeding with our bosonization solution, we linearize the $c$-electron spectrum about the two Fermi points and define left- and right-moving fermion fields $c_{\nu j}, \nu=$ $L(-), R(+)$, respectively, as subscript (otherwise). The chiral density operators $\rho_{\nu}(k)=\sum_{k^{\prime}} c_{\nu k^{\prime}+k}^{\dagger} c_{\nu k^{\prime}}$ obey the standard Luttinger commutators $\left[\rho_{\nu}(k), \rho_{\nu^{\prime}}\left(k^{\prime}\right)\right]_{-}=$ $\delta_{\nu, \nu^{\prime}} \delta_{k,-k^{\prime}} \nu k L / 2 \pi$ for a system of size $L \gg a$, the lattice constant. The dual Bose fields are constructed in terms of the $\rho_{\nu}(k)[9]$ :

$$
\begin{aligned}
& \phi\left(x_{j}\right)=-i \sum_{\nu} \sum_{k \neq 0} \frac{\pi}{k L} \rho_{\nu}(k) \Lambda_{\alpha}(k) e^{i k x_{j}}, \\
& \theta\left(x_{j}\right)=i \sum_{\nu} \sum_{k \neq 0} \nu \frac{\pi}{k L} \rho_{\nu}(k) \Lambda_{\alpha}(k) e^{i k x_{j}} .
\end{aligned}
$$

The cutoff function $\Lambda_{\alpha}(k)$ appearing in Eqs. (2) and (3) satisfies the conditions $\Lambda_{\alpha}(k) \approx 1$ for $|k|<\frac{\pi}{\alpha}$ and $\Lambda_{\alpha}(k) \approx$ 0 otherwise. $\Lambda_{\alpha}(k)$ enforces the finite minimum wavelength $\alpha>a$ of the bosonic density fluctuations $\rho_{\nu}(k)$ : Taking into account this wavelength limit is essential for preserving the lattice structure of the FKM [9]. Since the Bose fields cannot "resolve" distances less than $\alpha$, the usual commutators are "smeared" over this length:

$$
\begin{gathered}
{\left[\phi\left(x_{j}\right), \theta\left(x_{j^{\prime}}\right)\right]_{-}=\frac{i \pi}{2} \operatorname{sgn}_{\alpha}\left(x_{j^{\prime}}-x_{j}\right),} \\
{\left[\partial_{x} \phi\left(x_{j}\right), \theta\left(x_{j^{\prime}}\right)\right]_{-}=-i \pi \delta_{\alpha}\left(x_{j^{\prime}}-x_{j}\right) .}
\end{gathered}
$$

Here $\operatorname{sgn}_{\alpha}(x)$ and $\delta_{\alpha}(x)$ are the $\alpha$-smeared sign and Dirac $\delta$ functions, respectively [10].

The chiral fermions are represented in terms of the Bose fields by the Mandelstam identity $c_{\nu j}=$ $\sqrt{A a / \alpha} \hat{F}_{\nu} \exp \left(-i \nu\left[\phi\left(x_{j}\right)-\nu \theta\left(x_{j}\right)\right]\right)$. The dimensionless normalization constant $A$ is dependent upon the form of 
$\Lambda_{\alpha}(k)$; the Klein factors $\hat{F}_{\nu}$ are "ladder operators" between subspaces of differing $c$-electron number. The bosonic form of the number operators is given by

$$
n_{j}^{c}=n_{0}^{c}-\frac{a}{\pi} \partial_{x} \phi\left(x_{j}\right)+\frac{A a}{\alpha} \sum_{\nu} \hat{F}_{\nu}^{\dagger} \hat{F}_{-\nu} e^{i 2 \nu \phi\left(x_{j}\right)} e^{-i 2 \nu k_{F} x_{j}} .
$$

The first term on the right-hand side, $n_{0}^{c}$, is the noninteracting $c$-electron concentration; the second term is the forward-scattering density fluctuation; the third term is the first order backscattering correction. Higher order corrections are usually neglected.

Our bosonization analysis requires that $n_{0}^{c} \neq 0$. For the sake of brevity, here we assume the symmetric case with equal $c$ and $f$ populations in the noninteracting limit, i.e., $n_{0}^{c}=n_{0}^{f}=\frac{1}{2} n$. For the VT problem, this pins the Fermi level at $\epsilon_{f}=-2 t \cos \left(k_{F} a\right) ; k_{F}=\pi n / 2 a$ is the Fermi momentum (note that $\epsilon_{f}$ is irrelevant to the $\mathrm{CP}$ ). Using the electron concentration condition, we also rewrite the Coulomb interaction up to a constant: $G \sum_{j} n_{j}^{c} n_{j}^{f}=$ $G \sum_{j} n_{j}^{c}\left(n_{j}^{f}-\frac{1}{2}\right)-\frac{1}{2} G \sum_{j} n_{j}^{f}$. It is now a simple matter to obtain the bosonized form of the Hamiltonian by substituting these identities into Eq. (1).

We apply a shift transformation on the $c$-electron bosonic fields $\hat{U}=\exp \left\{i \frac{G a}{\pi v_{F}} \sum_{j}\left(n_{j}^{f}-\frac{1}{2}\right) \theta\left(x_{j}\right)\right\}$, where $v_{F}=$ $2 t a \sin \left(k_{F} a\right)$ is the $c$-electron Fermi velocity. This transformation rotates the basis of the Hilbert space so that the $c$ electrons are explicitly coupled to the $f$ orbitals. Introducing a pseudospin- $\frac{1}{2}$ representation for the $f$-electron occupation at site $j, n_{j}^{f}-\frac{1}{2}=\tau_{j}^{z}$, we write the transformed Hamiltonian

$$
\begin{aligned}
\hat{U}^{\dagger} \mathcal{H}_{\mathrm{FKM}} \hat{U}= & \frac{v_{F} a}{2 \pi} \sum_{j}\left\{\left(\partial_{x} \phi\left(x_{j}\right)\right)^{2}+\left(\partial_{x} \theta\left(x_{j}\right)\right)^{2}\right\} \\
& +\frac{1}{2} G(n-1) \sum_{j} n_{j}^{f} \\
& -\frac{G^{2} a^{2}}{2 \pi v_{F}} \sum_{j, j^{\prime}} \tau_{j}^{z} \delta_{\alpha}\left(x_{j}-x_{j^{\prime}}\right) \tau_{j^{\prime}}^{z} \\
& -\frac{2 G A a}{\alpha} \sum_{j} \tau_{j}^{z} \cos \left(2 \left\{\phi\left(x_{j}\right)-\mathcal{K}_{\alpha}\left(x_{j}\right)\right.\right. \\
& \left.\left.-\left[k_{F}+\frac{\pi}{2 a}\right] x_{j}\right\}\right),
\end{aligned}
$$

where $\quad \mathcal{K}_{\alpha}\left(x_{j}\right)=\mathcal{S}_{\alpha}\left(x_{j}\right)+\mathcal{L}_{\alpha}\left(x_{j}\right), \quad \mathcal{S}_{\alpha}\left(x_{j}\right)=\frac{\pi}{2} \times$ $\sum_{n=1}^{\infty}\left[\operatorname{sgn}_{\alpha}\left(x_{j+n}-x_{j}\right)-1\right]\left(\tau_{j+n}^{z}-\tau_{j-n+1}^{z}\right), \quad$ and $\mathcal{L}_{\alpha}\left(x_{j}\right)=\frac{\pi}{2}\left(\frac{G a}{\pi v_{F}}-1\right) \sum_{n=1}^{\infty} \operatorname{sgn}_{\alpha}\left(x_{j+n}-x_{j}\right)\left(\tau_{j+n}^{z}-\tau_{j-n}^{z}\right)$. $\mathcal{S}_{\alpha}\left(x_{j}\right)$ is a measure of the short-range behavior of the pseudospins, subtracting the $\tau^{z}$ within $\alpha$ to the left of $x_{j}$ from the $\tau^{z}$ within $\alpha$ to the right; $\mathcal{L}_{\alpha}\left(x_{j}\right)$ probes the longrange behavior, subtracting the $\tau^{z}$ more than $\alpha$ to the left of $x_{j}$ from the $\tau^{z}$ more than $\alpha$ to the right [11]. We retain all terms produced by the canonical transform in Eq. (7).
The removal of the forward-scattering Coulomb interaction introduces the important new term

$$
\mathcal{H}_{\mathrm{int}}=-\frac{G^{2} a^{2}}{2 \pi v_{F}} \sum_{j, j^{\prime}} \tau_{j}^{z} \delta_{\alpha}\left(x_{j}-x_{j^{\prime}}\right) \tau_{j^{\prime}}^{z} .
$$

The origin of this interaction is the delocalization of the $c$ electrons below scales less than $\alpha>a$. A $c$ electron spread over several lattice sites carries the same charge over these sites: Due to the $c-f$ repulsion $G$, this favors empty underlying $f$ orbitals. Although such "segregation" is known to occur in the CP $[3,5]$, the derivation of the responsible effective interaction is a new achievement in the history of the model. The $\alpha$-smeared $\delta$ function in Eq. (8) implies that the interaction is short-ranged; we therefore approximate the interaction $\mathcal{H}_{\text {int }} \approx-\mathcal{J} \sum_{j} \tau_{j}^{z} \tau_{j+1}^{z}, \quad \mathcal{J}=$ $G^{2} a^{2} \delta_{\alpha}(a) / \pi v_{F}$.

The other terms in Eq. (7) involving the pseudospins are a constant and a site-dependent field. The former is important only in the VT: Together with the first term in Eq. (7), this determines the distribution of the electron population across the two orbitals. The site-dependent field arises from the $2 k_{F}$ backscattering of the $c$ electrons off the $f$ orbitals. This leads to the known LRO phases, in analogy to the Peierls state [4]. It is clear from Eq. (7) that the LRO dominates the segregation at weak coupling $G \ll t$; increasing $G$, however, Eq. (8) eventually causes the system to segregate (the SEG phase).

The competition between the SEG and LRO phases can be simply studied within the framework of our bosonization approach: Since the only coupling in Eq. (7) between the Bose fields and the $\tau$ pseudospins is in the backscattering term, by replacing $\phi$ with a suitably chosen expectation value we obtain an effective Hamiltonian $\mathcal{H}_{\text {eff }}$ for the $f$ occupation only. Exact diagonalization calculations on 3200-site chains reveals that, within the SEG phase, the $c$ electrons are at their Luttinger liquid fixed point for all $G$ [12]. The choice $\left\langle\phi\left(x_{j}\right)\right\rangle=0$ is therefore valid across the phase diagram [9]. Substituting this into Eq. (7), we find

$$
\begin{aligned}
\mathcal{H}_{\mathrm{eff}}= & -\mathcal{J} \sum_{j} \tau_{j}^{z} \tau_{j+1}^{z}-\frac{2 G A a}{\alpha} \sum_{j} \tau_{j}^{z} \cos \left(2 \left\{\mathcal{K}_{\alpha}\left(x_{j}\right)\right.\right. \\
& \left.\left.+\left[k_{F}+\frac{\pi}{2 a}\right] x_{j}\right\}\right) .
\end{aligned}
$$

Below we present a detailed analysis of $\mathcal{H}_{\text {eff }}$.

Since the $c$ and $f$ populations are fixed in the CP, Eq. (9) by itself fully describes the system close to the SEG-LRO boundary. To determine this line, the site dependence of the longitudinal field must be known; in particular, we examine $\mathcal{S}_{\alpha}\left(x_{j}\right)$ and $\mathcal{L}_{\alpha}\left(x_{j}\right)$. Within the SEG phase, the $f$ electrons are arranged into a single block [5]. The magnitude of $\mathcal{L}_{\alpha}\left(x_{j}\right)$ reaches a maximum at the edge of this block, increasingly linearly as the edge is approached from either side. In contrast, $\mathcal{S}_{\alpha}\left(x_{j}\right)$ vanishes everywhere except 
in the vicinity of this edge. The longitudinal field, thus, has the general form $h_{j}^{z} \propto \cos (\omega j+\phi), \omega$ and $\phi$ constants.

The periodicity $(\omega)$ of $h_{j}^{z}$ determines the critical Ising coupling [13]: For $G \ll t$, we find the critical line $G_{c} a=$ $v_{F} \sqrt{2 \pi A / \alpha} \delta_{\alpha}(a)$. The $f$-electron configuration acts as an applied scattering potential $G\left\langle\tau_{j}^{z}\right\rangle$ for the $c$ electrons [12]. Below the average interparticle separation $\sim k_{F}^{-1}$, the $c$ electrons scatter independently: For $n \ll 1$, the low-energy $(E=0)$ wave functions $\psi$ therefore obey the Schrödinger equation $\partial_{x}^{2} \psi(x)=G m\left\langle\tau_{x}^{z}\right\rangle \psi(x)$ in the continuum limit $(m$ is the bare electron mass). From elementary quantum mechanics, we find plane wave solutions over unoccupied orbitals $\left(\left\langle\tau_{x}^{z}\right\rangle=-\frac{1}{2}\right)$ and exponentially decaying solutions with characteristic length $\zeta \propto \sqrt{t / G}$ over occupied orbitals $\left(\left\langle\tau_{x}^{z}\right\rangle=\frac{1}{2}\right)$ [14]. $\zeta$ clearly corresponds to $\alpha$ in the boson theory; for small $G_{c}$ Taylor expanding $\delta_{\alpha}(a)$ in the critical line equation gives $G_{c} a / v_{F}=\sqrt{2 \pi^{2} A}\left(1+a^{2} / 2 \alpha^{2}+\right.$ $\cdots)$, implying a linear relationship between $G_{c} a / v_{F}$ and $G_{c}$. This is confirmed at intermediate coupling by examining the numerical data of Gajek et al., with our fit giving $A=1 / 32 \pi^{2}, \alpha=a / 2 \sqrt{G_{c}}$, and a critical line $G_{c} / t=$ $0.5 \sin (n \pi / 2) /(1-\sin (n \pi / 2))$ as plotted in Fig. 1. This is the first analytic expression for the SEG-LRO boundary valid at weak coupling.

Moving on to the VT problem, it is clear from Eq. (7) that the Coulomb interaction will shift the $f$ level downwards, emptying the $c$ band. The depletion of the $c$-electron population causes segregation to occur at a smaller value of $G$ than in the CP; the new boundary can be calculated self-consistently from the available numerical results [3]. Using the same curve-fitting technique as for the $\mathrm{CP}$, we find excellent agreement with the data for $G_{c} / t=0.35 \sin (n \pi / 2) /[1-\sin (n \pi / 2)] \quad(A \approx 1.55 \times$ $\left.10^{-3}, \alpha \approx 0.418 a / \sqrt{G_{c}}\right)$; see Fig. 1. Further increasing $G$ above the critical value $G / t=4(1-\cos (n \pi / 2)) /(1-$ $n$ ), the $f$ level lies below the $c$-electron band edge and so all electrons have $f$ character. The absence of $c$ electrons to mediate the segregating interaction implies that here any $f$ configuration is the ground state.

We extend our analysis to the physically interesting limit $V \ll t$ of the QFKM. Bosonizing and carrying out the canonical transform, the hybridization adds the term

$$
\hat{U}^{\dagger} \mathcal{H}_{\mathrm{hyb}} \hat{U}=4 V \sqrt{\frac{A a}{\alpha}} \sum_{j} \tau_{j}^{x} \cos \left\{\mathcal{K}_{\alpha}\left(x_{j}\right)+\left[k_{F}+\frac{\pi}{2 a}\right] x_{j}\right\}
$$

to the effective pseudospin Hamiltonian Eq. (9). We have used a generalized Jordan-Wigner transformation to combine the $f$-electron operators and the Klein factors into pseudospins $\tau_{j}^{+}=f_{j}^{\dagger} \hat{F}_{\nu} e^{-i \nu \pi x_{j} / 2 a} \exp \left(-i \frac{\nu \pi}{2} \sum_{j^{\prime}} \operatorname{sgn}\left(x_{j^{\prime}}-\right.\right.$ $\left.\left.x_{j}\right)\left(n_{j^{\prime}}^{f}-\frac{1}{2}\right)\right)$. The resulting effective pseudospin Hamiltonian is easily recognized as the Ising model in sitedependent longitudinal $\left(h_{j}^{z}\right)$ and transverse $\left(h_{j}^{x}\right)$ fields.

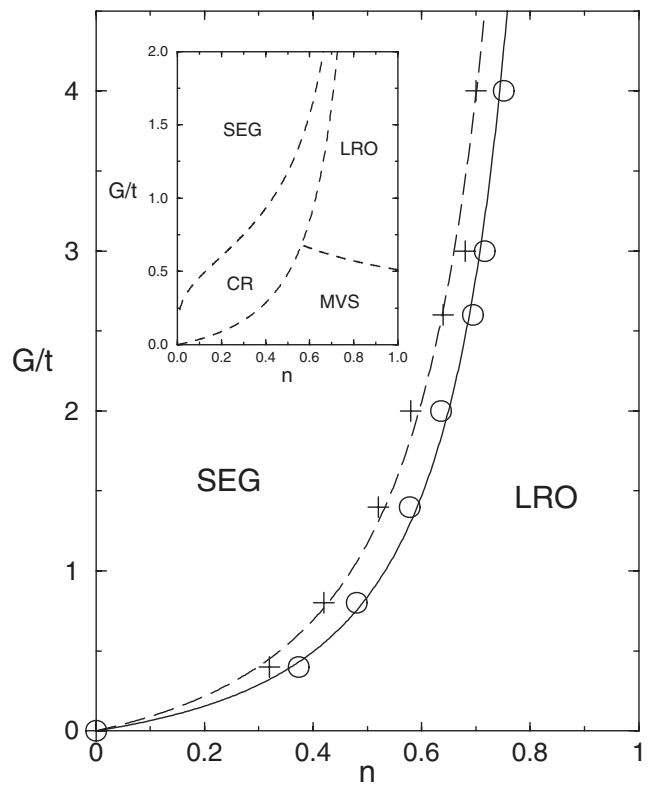

FIG. 1. Ground-state phase diagram for the symmetric FKM below half-filling for the CP (dashed line) and VT problems (solid line). The numerical data points (circles for VT, plus sign for CP) are due to Gajek et al. [3]. Inset: The phase diagram for the QFKM. Phases are as described in text.

Although we are not aware of any systematic study of this Hamiltonian, we can nevertheless deduce important aspects of its physical behavior. The two limits $t \sim G \gg V$ and $G \ll V$ are easiest to discuss. In the former case, the hybridization does not radically modify the VT physics. Where the longitudinal field dominates, we can discard the Ising interaction so that the pseudospins track the longitudinal and transverse fields; clearly, the transverse field will be important only where the longitudinal field is small. This will "smear" the crystalline arrangement but leave the basic picture of a density wave intact. Where the Ising interaction dominates, the hybridization will stabilize the SEG phase as quantum fluctuations maintain a nonzero $c$-electron population.

For $G \ll t$, the quantum fluctuations produced by the hybridization dominate, fundamentally altering the behavior of the system. Discarding the Ising interaction which is irrelevant for most of this regime, we arrive at the same trivial model of pseudospins tracking longitudinal and transverse fields as in the $G \sim t$ LRO phase. Here, however, the transverse field is generally much larger than the longitudinal field: The pseudospins will be mostly ordered along the $x$ axis with $\left\langle\tau_{j}^{x}\right\rangle \approx \frac{1}{2} \operatorname{sgn}\left(h_{j}^{x}\right)$. In terms of the $f$ occupation, this implies that $\left\langle f_{j}^{\dagger} c_{j}\right\rangle \neq 0$, with intermediate occupation of each $f$ orbital $0<\left\langle n_{j}^{f}\right\rangle \approx \frac{1}{2} n$. The longitudinal field produces only small deviations from this homogeneous distribution of electron density. This is clearly a mixed-valence state (MVS).

Our pseudospin model allows us to connect these two limits for the first time: For $V=0.05 t$, we present the QFKM's phase diagram as the inset in Fig. 1. Close to 
half-filling, the Ising interaction is never dominant, so the model of $\tau$ pseudospins tracking the longitudinal and transverse fields is valid for any $G$. In Fig. 1, we define an MVS-LRO boundary by equating the magnitude of the transverse and longitudinal fields. For small $n$, however, the longitudinal field is negligible; discarding this term from $\mathcal{H}_{\text {eff }}$, we find that a transverse field Ising model (TFIM) describes the $f$-orbital occupation. Although the TFIM has an order-disorder transition [15], the shifting of the $f$ level lifts the system from criticality and so there is a crossover regime (CR) between the SEG and MVS phases.

The details of the CR physics are controlled by the site dependence of the transverse field Eq. (10). Near the SEG phase, we can use the same values for $\mathcal{S}_{\alpha}\left(x_{j}\right)$ and $\mathcal{L}_{\alpha}\left(x_{j}\right)$ as in the CP analysis; for MVS behavior, both these objects vanish. We thus assume a periodic transverse field within the CR. In general, the periodicity is incommensurate with the lattice: Numerical studies [16] reveal this to be qualitatively identical to a random variation of the transverse field. The random TFIM has been studied in depth by Fisher [15] using a real-space renormalization group treatment. To relate Fisher's results to the CR, we assume an effective Hamiltonian $\mathcal{H}_{\mathrm{CR}}=-\mathcal{J} \sum_{j} \tau_{j}^{z} \tau_{j+1}^{z}+\sum_{j} h_{j}^{x} \tau_{j}^{x}$, where the values of $h_{j}^{x}$ are drawn randomly from the cosine distribution $\rho(h) d h=(C \pi)^{-1} \sqrt{1-(h / C)^{2}}, C=$ $4 V \sqrt{A a / \alpha}$. For the CR, $A$ and $\alpha$ are as in the SEG phase; we thus use the values from the $V=0$ VT problem.

Lowering $G$ from the SEG phase, the CR is reached at $\mathcal{J}_{c 1}=\max \left|h_{j}^{x}\right|$ : For $\mathcal{J}<\mathcal{J}_{c 1}$, the Ising coupling is not greater than the magnitude of the transverse field everywhere on the lattice. The rare regions where $\left|h_{j}^{x}\right|>\mathcal{J}$ break the SEG ground state up into randomly distributed large clusters of zero and full $f$ occupancy. The system nevertheless retains the character of the SEG phase so long as $\mathcal{J}>\mathcal{J}_{c 2}=\overline{\left|h_{j}^{x}\right|}$, the average value of $\left|h_{j}^{x}\right|$ across the lattice. This is reflected by the mean $f$ - $f$ correlations $\overline{\left\langle n_{j}^{f} n_{j+x}^{f}\right\rangle}$ which decay exponentially to $\left(n^{f}\right)^{2}>\left(n_{0}^{f}\right)^{2}$ with correlation length $\xi \sim \exp \left\{-2\left[\ln \left(\mathcal{J}_{c 2} / \mathcal{J}\right)\right]^{2}\right\}$.

Decreasing $\mathcal{J}$ below $\mathcal{J}_{c 2}$, the character of the CR reverses, as here $\mathcal{J}<\left|h_{j}^{x}\right|$ over most of the lattice. Of the SEG state, only rare clusters remain, embedded in a valence-fluctuating background. This MVS-like region of the CR is characterized by a different functional form for the correlation length $\xi \sim\left[\ln \left(\mathcal{J}_{c 2} / \mathcal{J}\right)\right]^{-2}$; the correlation functions decay exponentially to $\left(n_{0}^{f}\right)^{2}$. The CR persists until $\mathcal{J}$ becomes comparable to the longitudinal field in Eq. (9): The system is then best described by the small- $G$ MVS. In Fig. 1, the upper boundary of the CR is derived from the condition $\mathcal{J}_{c 1}=\max \left|h_{j}^{x}\right|$ and is given by $G_{c 1}=$ $2\left[\left\{V v_{F} \pi / a \delta_{\alpha}(a)\right\} \sqrt{A / a \alpha}\right]^{1 / 2}$; the lower boundary is the critical line $G_{c}$ as the $V=0$ VT problem. We extend the curve $G_{c 1}(n)$ to $G>t$ using the VT result for $\alpha$.

The distinguishing feature of the $\mathrm{CR}$ is the randomly distributed clusters of integral $f$ valence. These clusters occur where the local $c$-electron density departs from the MVS average, determining the occupation of the underlying $f$ orbitals via the forward-scattering Coulomb interaction [i.e., Eq. (8)]. This coupling of the $c$ - and $f$-electron densities is an electronic polaron. Studying the QFKM, Liu proposed that electronic polaron effects appeared intermediate between mixed- and integral-valence phases [7]. Since bosonization treats the forward-scattering exactly [9], our calculation rigorously confirms Liu's scenario.

In summary, we have presented the results of a novel theoretical study of the 1D FKM. Using a nonperturbative approach, we have uncovered the physical mechanisms responsible for the known $f$-electron ordering. We derive an effective Hamiltonian for the occupancy of the $f$ orbitals which predicts the SEG-LRO transition. We also study the QFKM for small hybridization $V \ll t$. For the first time, we can accurately interpolate between the wellknown CP and MVS limits: At intermediate coupling, we find a crossover regime where electronic polaron effects are of importance.

P.M.R.B. acknowledges useful discussions with C.D. Batista and J. E. Gubernatis.

[1] L. M. Falicov and J. C. Kimball, Phys. Rev. Lett. 22, 997 (1969).

[2] T. Kennedy and E. H. Lieb, Physica (Amsterdam) 138A, 320 (1986).

[3] Z. Gajek, J. Jędrzejewski, and R. Lemański, Physica (Amsterdam) 223A, 175 (1996).

[4] J. K. Freericks, Ch. Gruber, and N. Macris, Phys. Rev. B 53, 16189 (1996).

[5] P. Lemberger, J. Phys. A 25, 715 (1992).

[6] J. M. Lawrence, P. S. Riseborough, and R. D. Parks, Rep. Prog. Phys. 44, 1 (1981).

[7] S. H. Liu, Phys. Rev. Lett. 58, 2706 (1987).

[8] T. Portengen, Th. Östreich, and L. J. Sham, Phys. Rev. Lett. 76, 3384 (1996).

[9] M. Gulácsi, Adv. Phys. 53, 769 (2004).

[10] We use $\Lambda_{\alpha}(k)=e^{-\alpha|k|}$ and so we have $\delta_{\alpha}(x)=\frac{1}{\pi} \frac{\alpha}{\alpha^{2}+x^{2}}$, $\operatorname{sgn}_{\alpha}(x)=\frac{2}{\pi} \arctan \left(\frac{x}{\alpha}\right)$.

[11] Using spin- $\frac{1}{2}$ identities, the argument of the cosine is considerably simplified. We retain the general form, however, as these cancellations cannot be made when hybridization is present.

[12] P. Farkašovský, Int. J. Mod. Phys. B 17, 4897 (2003).

[13] C. Sire, Int. J. Mod. Phys. B 7, 1551 (1993).

[14] L. I. Schiff, Quantum Mechanics (McGraw-Hill, New York, 1955).

[15] D. S. Fisher, Phys. Rev. B 51, 6411 (1995).

[16] I. I. Satija, Phys. Rev. B 41, 7235 (1990). 